\title{
LA PROBLEMÁTICA DE LA EDUCACIÓN SUPERIOR Y EL RETO DE LA CALIDAD
}

\author{
MARTHA PÉREZ CONTRERAS DE CHEN ${ }^{1}$
}

RESUMEN: El presente artículo plantea como objetivo describir brevemente la problemática de la educación superior y el reto de la calidad. Para ello se abordan los principales desafíos que abordan las universidades principalmente relacionados con la calidad, así como los conceptos que más se relacionan con ella. De igual forma se realiza un recorrido por las aproximaciones al concepto de la calidad desde diferentes perspectivas multidimensionales, a partir de una visión intrínseca y extrínseca y como se complementa con otros abordajes sobre dicho concepto.

PALABRAS CLAVE: educación superior; calidad; retos de la educación; problemática educativa.

\section{The problematic of higher education and the challenge of quality}

ABSTRACT: The objective of the following article is to briefly describe the problems of higher education and the challenges of quality assurance. To this end, it addresses the main challenges that universities face, mainly related to quality, as well as the concepts that are most closely related to it. Likewise, a review is made of the approaches to the concept of quality from different multidimensional perspectives, from an intrinsic and extrinsic vision and how it is complemented with other approaches to this concept.

KEY WORDS: higher education; quality; educational challenges; educational problems.

\section{INTRODUCCIÓN}

Al pensar en la importancia de la calidad en todo sistema de educación superior y la necesidad de trabajar para mejorarla, es imposible no traer a la mente al Doctor Pedro Morales, S.J., quien ha dedicado toda su trayectoria profesional a buscar siempre la excelencia en las distintas universidades en

1 Universidad Rafael Landívar (Guatemala). Correo electrónico: mdchen@url. edu.gt. 
las que ha colaborado. Muestra de ello es que además de incidir en las tres funciones sustantivas de docencia, investigación y proyección, dentro de las múltiples investigaciones que ha realizado se encuentra la temática de la calidad. Sabiendo que la calidad es un eje fundamental que debe estar presente en todo sistema de educación superior y que es indispensable su evaluación de forma continua, es necesario tenerla presente como eje transversal en todas las discusiones educativas. Para Roa (2003) es la propia universidad la que debe tomar la iniciativa de establecer modelos y mecanismos de autorregulación que la comprometa en el cumplimiento de sus funciones sustantiva, sus objetivos y su misión. Las universidades requieren adicionalmente a los mecanismos internos de calidad, estar inmersas en sistemas nacionales o internacionales de aseguramiento de la calidad, que las retroalimenten desde una visión externa sobre su quehacer.

En su artículo titulado ¿Qué significa enseñar e investigar con calidad y equidad en la Educación Superior? Morales (2001) plantea que es necesario analizar la calidad de los componentes clave que conforman las universidades. Hablar de calidad significa:

- Enfocarse menos en la calidad de la enseñanza para enfatizar la importancia de la calidad del aprendizaje.

- Comprender que la calidad del aprendizaje está relacionada en buena medida con la calidad de la relación entre el profesor y sus alumnos.

- Que la calidad del aprendizaje no depende tanto de qué se estudia como de cómo se estudia.

- Que para hablar de calidad en la educación superior se requiere trabajar intensamente en la evaluación, en el currículo intencional pero también en el currículo oculto, así como en la evaluación de la evaluación.

- Que no es posible perder de vista la relación entre calidad, formación y satisfacción del profesorado.

- Relacionar la calidad y la equidad.

A partir de estas afirmaciones, son muchos los autores que han profundizado en ellas y a su vez han analizado el tema de la calidad en la educación superior. A continuación, se busca recoger los principales aportes en esta temática.

El presente artículo pretende ser un pequeño homenaje a un profesional que ha dedicado su vida a trabajar por la calidad en el contexto de la educación superior, convirtiéndose en un referente de lo que significa ser un educador. Gracias por la huella que ha dejado en todos los que hemos tenido el privilegio de conocerlo Padre Pedro Morales, S.J. 


\section{LA PROBLEMÁTICA DE LA EDUCACIÓN SUPERIOR Y EL RETO DE LA CALIDAD}

La percepción del deterioro de la calidad de la formación que se imparte en las instituciones de educación superior, se debe en buena medida a la proliferación de instituciones que no reúnen los requisitos mínimos para ofrecer un trabajo académico superior, de acuerdo a Tünnermann (1999); identificando también un bajo nivel académico de las plantas docentes, poca preparación y la utilización de métodos de enseñanza centrados en la transmisión de conocimientos y acumulación de información. Desde esta perspectiva Brunner (2003) señala que en 1950 no existían más de 75 universidades en América Latina y en la actualidad se cuenta con más de 5,000. Plantea que la educación superior ha sufrido en la última mitad del siglo una transformación sin precedentes, que tiene que ver con cuatro situaciones concretas: a) la proliferación de las instituciones y su diversidad; b) la participación creciente del sector privado en la educación; c) la ampliación del cuerpo docente con calidades distintas y d) el aumento en el número de graduados con características heterogéneas.

Orozco Silva (2010) al igual que Brunner identifica una serie de factores que han afectado el funcionamiento de las instituciones de educación superior y que requiere de ellas cambios que les permita adaptarse a los nuevos tiempos que estamos viviendo; cuestionando incluso si no sería necesario un replanteamiento de su misión. En sus investigaciones determina como factores de la crisis que afrontan las universidades los siguientes: a) la demanda creciente de la educación superior que ha hecho que las universidades prioricen la docencia y educación continua, dejando en segundo plano su función sustantiva de investigación; b) las nuevas demandas de los mercados laborares que exigen una educación más profesionalizante; c) las nuevas formas de producción y gestión del conocimiento que hacen necesario que las universidades trabajen en redes y cambien su estructura de funcionamiento; d) la creación de espacios de educación superior, que establezcan criterios comunes; y e) el creciente interés del sector externo en las instituciones educativas que buscan alianzas de beneficio mutuo.

Ante esta problemática ya no son únicamente las universidades las que se preocupan por la calidad de la educación, sino que diversas instituciones y organismos internacionales han manifestado su interés, realizando estudios exploratorios que concluyen sobre la relevancia de abordar este tema. De acuerdo a Rodríguez (2013) los principales hallazgos son:

- La necesidad del involucramiento de las autoridades universitarias que propicien la implantación de una cultura de calidad. 
- La identificación de factores que permitan garantizar la calidad de la enseñanza, como elemento clave dentro de las universidades, reconociéndola como algo dinámico y en constante evolución.

- La importancia que tiene que las instituciones de educación superior sean reconocidas como proveedoras de enseñanza de calidad.

- La influencia que ejercen los estudiantes, que cada vez más exigen garantía de que sus estudios les darán los conocimientos necesarios para su inserción laboral.

Ardila (2011) acuña un concepto importante y que merece la pena ser tomado en cuenta, tanto en la comprensión de la calidad educativa en el ámbito de la educación superior, como en el diseño de los sistemas de gestión de la calidad. Desde su perspectiva «la calidad de la educación superior se encuentra en un estadio que podemos llamarlo de transición, entre la concepción de calidad como responsabilidad del estado y la concepción de calidad como responsabilidad de todos; entre el control y vigilancia como únicas estrategias garantes de su presencia y la construcción colegiada desde perspectivas y enfoques transdisciplinares» (p. 49).

La UNESCO (1998) en el proyecto de declaración mundial sobre la educación superior en el siglo XXI: visión y acción, establece que:

La transformación y expansión de la educación superior, la mejora de su calidad y pertinencia y la manera de resolver las principales dificultades que la acechan exige la firme participación no solo del gobierno e instituciones de educación superior, sino también de todas las partes interesadas, comprendidos los estudiantes y sus familias, los profesores, el mundo de los negocios y la industria, los sectores público y privado de la economía, los parlamentos, los medios de comunicación, la comunidad, las asociaciones profesionales y la sociedad, y exigen igualmente que las instituciones de educación superior asuman mayores responsabilidades para con la sociedad y rindan cuentas de la utilización de los recursos públicos y privados, nacionales e internacionales. (p. 89)

\section{LA CALIDAD EDUCATIVA Y OTROS CONCEPTOS RELACIONADOS}

La UNESCO señala que, aunque en la última década se han llevado a cabo esfuerzos por mejorar la calidad, se debe trabajar también en la equidad en el acceso a la educación superior, no solo analizándola como la posibilidad de ingresar al sistema educativo, sino también de culminar los estudios y por ende de alcanzar los objetivo inicialmente establecidos (Conferencia mundial sobre la educación superior, 2009). 
El tema de la calidad y su relación con la equidad ha sido abordado por varios autores que coinciden en que una no puede ser analizada sin considerar a la otra. Garbanzo (2007) al igual que la UNESCO abordan como inseparables los términos de calidad educativa y equidad educativa. Plantea que la calidad es un concepto en evolución, que se ha venido modificando a lo largo del tiempo, pasando de ser cuantitativo a mucho más cualitativo e integral. Se establece entonces que no puede hablarse de calidad en la educación sino existen programas de equidad, que posibiliten el acceso al sistema de educación superior. La calidad implica que la educación llegue a toda la población en igualdad de condiciones y que brinde atención diferenciada para responder a las necesidades concretas de cada comunidad.

Arrien (1996) lo planteó desde hace aproximadamente 20 años, identificando problemas en el quehacer universitario, que resume con un desajuste entre su naturaleza y su forma de funcionar. Dentro de la discusión introdujo dos variables adicionales que son la pertinencia y la eficiencia. Desde su perspectiva la calidad siempre se entrelaza con la equidad, pertinencia y eficiencia, ya que su funcionamiento óptimo daría como resultado un desempeño de calidad en el sistema universitario que abarcaría a los programas académicos, los servicios, los procesos, los resultados y su impacto en la sociedad. Continúa expresando que la calidad debería buscar como fin último el desarrollo en el aprendizaje significativo e integral de las personas, permitiendo que incrementen conocimientos, habilidades, actitudes y valores que les permitan ser mejores ciudadanos, capaces de participar activamente en la construcción de una mejor sociedad. La calidad educativa entonces debe prestar especial interés en desarrollar competencias como «aprender a ser», «aprender a actuar y hacer», «aprender a convivir», «aprender a aprender», por mencionar algunas.

Retomando la relación de la calidad con la pertinencia, para Arrien una educación de calidad es aquella que responde a las dimensiones sociales, culturales e históricas de forma adecuada y creativa en un contexto específico. En cuanto a la equidad indica que es el derecho de toda persona a la educación, pero no la limita al ingreso al sistema educativo, sino como la responsabilidad de asegurar igualdad de condiciones en los procesos de aprendizaje; permitiendo de esta manera el éxito en la culminación de los estudios. Por último, al referirse a la eficiencia universitaria la concibe como «la capacidad práctica de hacer efectivas la calidad, pertinencia y equidad. Esto significaría, hacer las cosas correctamente y hacer lo correcto, es decir, lo que le compete por naturaleza de universidad» (p. 5).

La calidad de la educación se compone de varios enfoques o dimensiones que se complementan entre sí (Toranzos, 1996). La calidad desde su 
perspectiva está compuesta por tres elementos indispensables: a) eficiencia, b) relevancia y c) calidad de los procesos. Desde la eficiencia se establece que una institución goza de calidad cuando los estudiantes aprenden lo que deben aprender lo que está definido en los planes de estudio y en el perfil de egreso. En otras palabras, que sean transformados en su paso por el sistema y que se logren los objetivos de aprendizaje. La dimensión de relevancia en el concepto de calidad es aquella que logra que los alumnos no solo aprendan, sino que aprendan lo que necesitan aprender para desarrollarse dentro de la sociedad y sus diversos ámbitos. Por último, la tercera dimensión es la que abarca la calidad de los procesos y los medios que se ofrecen para optimizar tanto el aprendizaje como la enseñanza. En esta variable se incluyen las instalaciones, el cuerpo docente, adecuados materiales de estudio y herramientas de apoyo a la educación. Si una de ellas está ausente, el sistema educativo no estaría en capacidad de construir procesos de gestión de la calidad.

Otras investigaciones identifican también que la calidad tiene elementos que la conforman o se relacionan con ella. Para Vizcarra, Boza y Monteiro (2011), no es suficiente al hablar de calidad vincularla con equidad, eficiencia, relevancia y calidad de los procesos como se mencionó anteriormente. Establecen que son cinco los componentes que se entrelazan con la calidad: a) pertinencia; b) eficiencia en el desempeño; c) eficacia; d) productividad; e) acreditación; f) aseguramiento y e) sustentabilidad.

La pertinencia en la calidad se entiende como la utilidad para cumplir con una función específica, o a la utilidad de lo que hace una institución para satisfacer las demandas externas. Al comparar el desempeño real con el esperado dentro de una institución universitaria surge el término de eficacia, que no es otra cosa que el grado en que dicha institución cumple con el propósito para la fue diseñada, sin que existan brechas entre el ser y del deber ser. Cuando el propósito se cumple con el mejor aprovechamiento de los recursos existentes, se introduce la eficiencia. Se percibe con más calidad aquella universidad que logra objetivos concretos en menor tiempo y con menos recursos. La combinación de eficacia y eficiencia da paso a la productividad, que es otro diferenciador de su calidad.

Continúan planteado los autores que también se encuentra presente como elemento de la calidad la acreditación, que consiste en su validación externa por pares académicos objetivos e independientes. Luego de esta validación se hace necesaria la implementación de sistemas permanentes que reflexionen sobre la calidad educativa y que sean reconocidos y validados por los actores educativos. Es entonces cuando surge el aseguramiento de la calidad entendido como mejora continua. Los juicios de calidad en un sistema de educación superior que consideran únicamente a la pertinencia, 
eficacia, eficiencia, productividad, acreditación y el aseguramiento de la calidad, se encuentran incompletos. Es necesario considerar como último elemento la sustentabilidad, que se comprende como el cumplimento de los propósitos educativos sin consecuencias internas o externas que afecten a la sociedad.

Tabla 1. Conceptos clave Relacionados con la calidad

\begin{tabular}{|c|c|c|c|c|c|}
\hline \multirow{2}{*}{$\begin{array}{c}\text { Conceptos } \\
\text { relacionados } \\
\text { con la calidad }\end{array}$} & \multicolumn{5}{|c|}{ Autores } \\
\hline & $\begin{array}{c}\text { Arrien } \\
1996\end{array}$ & $\begin{array}{c}\text { Toranzos } \\
1996\end{array}$ & $\begin{array}{c}\text { UNESCO } \\
1998\end{array}$ & $\begin{array}{c}\text { Garbanzo } \\
2007\end{array}$ & \begin{tabular}{|c} 
Vizcarra, Boza \\
y Monteiro \\
2011
\end{tabular} \\
\hline 1. Equidad & & & $\mathrm{X}$ & $\mathrm{X}$ & \\
\hline 2. Pertinencia & $\mathrm{X}$ & & & & $\mathrm{X}$ \\
\hline 3. Relevancia & & $\mathrm{X}$ & & & \\
\hline $\begin{array}{l}\text { 4. Calidad de } \\
\text { procesos }\end{array}$ & & $\mathrm{X}$ & & & \\
\hline 5. Eficacia & & & & & $\mathrm{X}$ \\
\hline 6. Eficiencia & $\mathrm{X}$ & $\mathrm{X}$ & & & $\mathrm{X}$ \\
\hline 7. Productividad & & & & & $\mathrm{X}$ \\
\hline 8. Acreditación & & & & & $\mathrm{X}$ \\
\hline $\begin{array}{l}\text { 9. Aseguramiento } \\
\text { de la calidad }\end{array}$ & & & & & $\mathrm{X}$ \\
\hline 10. Sustentabilidad & & & & & $\mathrm{X}$ \\
\hline
\end{tabular}

Fuente: elaboración propia a partir de Arrien, Toranzos, UNESCO, Garbanzo y Vizcarra.

La eficiencia es el elemento incluido con mayor frecuencia en el desarrollo de los autores presentados; seguidos por la equidad y la pertinencia. Haciendo entonces que el logro de los propósitos y objetivos de las instituciones, acompañados de la optimización de los recursos que se emplean, sean importantes en los planteamientos del logro de la calidad en la educación superior.

Las discusiones sobre la calidad en la educación superior y sus implicaciones parecieran no tener fin, ya que cada abordaje plantea una visión 
distinta basada en experiencias propias y contextos diversos. Acertadamente Giraldo, Abad y Díaz (2003) plantean que la calidad puede tener distintos significados para una misma persona o institución en situaciones y momentos diferentes; reafirmando también su perspectiva multidimensional y sus diversas interpretaciones, de acuerdo a la formación de las personas, diversidad de pensamientos, y enfoques. Por lo tanto, no puede ser definida en términos absolutos. Al igual que con la belleza, esta dependerá de la óptica con la que se analice. Mencionan que, en una institución educativa, una estrategia de mejoramiento debe partir de una clara definición operativa de lo que se entiende por calidad, tomándola como punto de referencia al momento de evaluar algún aspecto específico del sistema educativo de la institución; tratando de establecer criterios e indicadores concretos que permitan la objetividad de dicha evaluación.

Con lo anterior su importancia no pierde sentido, ya que muchos expertos coinciden en la necesidad de un análisis serio y profundo que conduzca a la reflexión de la calidad en los sistemas de educación superior.

\section{APROXIMACIONES AL CONCEPTO DE CALIDAD}

Indiscutiblemente debe abordarse el tema de la calidad, pero ¿Qué se entiende por calidad? ¿Cómo podemos comprenderla? Hablar sobre calidad no es una tarea fácil y menos aún intentar definirla, ya que existen distintas aproximaciones y maneras diversas de interpretarla. Al desarrollar el tema de calidad en la educación, la gran mayoría comprende su significado, pero son pocos lo que se atreven a explicar de qué se trata (Valdés, 2005).

«El concepto de calidad se desarrolló en el mundo empresarial hasta convertirse en la actualidad en una necesidad estratégica y en un arma para sobrevivir en mercados altamente competitivos» (Vizcarra, Boza y Monteiro, 2011, p. 292). De ahí migró al ámbito de la educación superior y a otros sectores sociales, debido a la necesidad de abordarla y de estableces sistemas de mejoramiento continuo. La concepción de la calidad se ve modificada por la injerencia de demandas de distintos sectores de la sociedad como: a) el estado; b) sectores sociales; c) sectores de desarrollo de ciencia y tecnología d) y de la empresa. 
Figura 1. Demandas como factores que modifican EL CONTENIDO DEL CONCEPTO DE CALIDAD

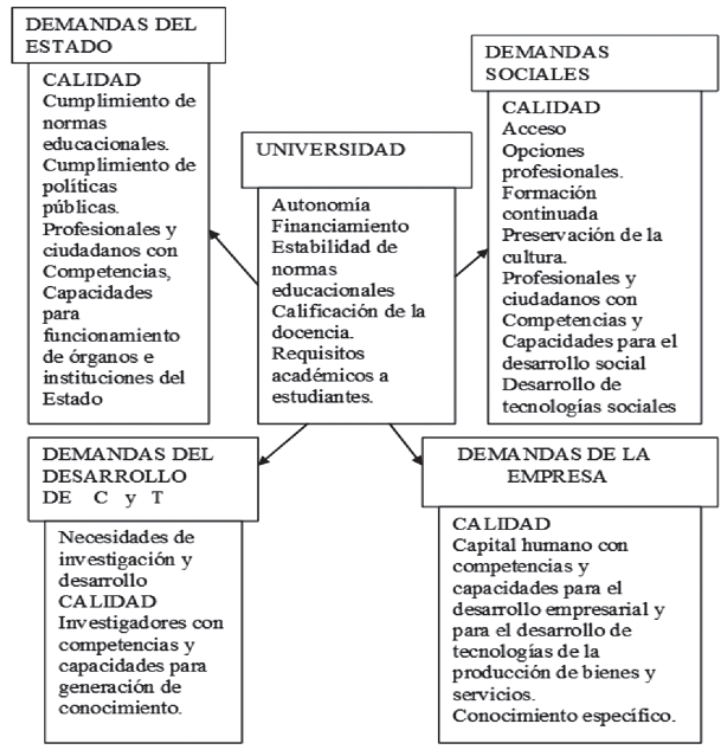

Fuente: Vizcarra, Boza y Monteiro (2011, p. 302).

\subsection{LA PERSPECTIVA MULTIDIMENSIONAL DE LA CALIDAD}

Existe una gran cantidad de definiciones como autores tratando de explicar de qué trata la calidad en la educación superior. Las discusiones concuerdan que no es posible llegar a una aproximación única, ya que es un concepto relativo. Rodríguez Espinar (2013) citando a Vroeijenstijn, menciona que la relatividad del concepto de calidad se debe a que los actores de la educación superior tienen intereses diferentes. Concibe a la calidad como un conjunto de cualidades o dimensiones; en cuanto es posible identificar que una universidad puede ser de «mayor calidad» en relación a una dimensión concreta, pero de menor calidad con relación a otra. Es por tanto indispensable que al momento de calificar se expliciten con toda claridad los criterios sobre los que se otorga dicho calificativo. Asimismo, Duk y Narvarte (2008) lo plantean como un concepto compuesto de distintos elementos. 
Reconocer su complejidad no justifica que no se discuta que elementos o características conforman la calidad de la educación, sino que abre paso a investigaciones que proponen definiciones de calidad multidimensionales. De igual forma permite analizar si es correcto que al identificar algún componente clave del sistema educativo y definirlo como de calidad, esto haga que el sistema como tal pueda ser considerado de la misma manera.

El Diccionario de la Real Academia Española (2005) define a la calidad como «conjunto de propiedades inherentes a una persona o cosa, que permiten valorarlas con respecto a otras de su misma clase o especie» (p. 242). Dando así amplitud a su concepción y la necesidad de establecer características que permitan la comparación.

Para UNESCO (1998) el concepto de calidad de la educación superior es pluridimensional y abarca todas las funciones, actividades y servicios que se ofrecen en las instituciones, tales como: programas académicos, proceso de enseñanza, desarrollo de investigación, el personal (académico y administrativo), los estudiantes, la infraestructura y los servicios. Su evaluación debería partir de procesos de autoevaluación interna validados por mecanismos externos o llevados a cabo por expertos independientes. Plantea que también sería prioritaria la creación de instancias nacionales que garanticen el buen desempeño de la educación superior. Define como componente importante de la calidad a la internacionalización, que permita definir estándares de comparación, que, por supuesto respeten las diferencias de los distintos contextos, pero que apoyen la movilidad de docentes y estudiantes, el desarrollo de investigaciones conjuntas y que faciliten compartir el conocimiento.

La Red Iberoamericana para la Acreditación de la Calidad en la Educación Superior RIACES (2004) coincide con que no hay acuerdo universal sobre lo que se entiende por calidad, pero que cada vez más se mide en relación a dos elementos: a) formación de las personas que terminan el programa y b) la capacidad de la institución para producir cambios que mejoren esa formación y planificación de ese cambio; mediante estrategias que propicien también la transformación institucional. Presenta la definición de calidad desde las siguientes vertientes (p. 20):

- Grado en el que un conjunto de rasgos diferenciadores inherentes a la educación superior cumple con una necesidad o expectativa establecida.

- Propiedad de una institución o programa que cumple los estándares previamente establecidos por una agencia u organismo de acreditación.

- La calidad implica la evaluación de la docencia, el aprendizaje, la gestión y los resultados obtenidos. El conjunto de todos estos elementos supone la calidad de una institución. 
El tema de calidad se ha venido discutiendo en todos los ámbitos imaginables que oscilan desde los empresariales, de producción, y sin duda alguna en los educativos. En pocas palabras en todos los aspectos de la vida. Bondarenko (2007) citando a Moreno, Hernández, Lengster, Moreno y Gonzáles afirma que luego de revisar la bibliografía de expertos que abordan el tema, se determinan por lo menos siete definiciones de la calidad:

1. Aptitud para el uso.

2. Satisfacción del cliente.

3. Conveniencia al uso o conveniencia al propósito.

4. Conformidad con los requisitos.

5. Un producto o servicio libre de defectos.

6. Capacidad para satisfacer las expectativas del consumidor.

7. Cumplimiento o superación de las expectativas del cliente a un costo que le represente valor.

A su vez, González y Espinoza (2008) citando a Días Sobrinho plantea que la calidad es:

Una construcción social, que varía según los intereses de los grupos de dentro y de fuera de las instituciones. Por ejemplo, los académicos les asignan importancia a los aspectos académicos (el conocimiento, los saberes); los empleadores a las competencias con que los estudiantes egresan y que les permiten integrarse al campo de trabajo; los estudiantes a la empleabilidad. Es necesario que esta construcción social sea adoptada y compartida y atraviese el quehacer de las funciones esenciales de la universidad (p. 4).

La calidad educativa según Buendía (2007), es el resultado de un sistema donde se articulan tres elementos clave: «el ser, el hacer y el deber ser de las instituciones de educación superior». Define también tres características que hacen realidad los elementos clave de la calidad:

- Coherencia de los programas que se ofrecen con las necesidades y las características del entorno.

- Eficacia planteada como la coherencia entre las actividades definidas por la institución para cumplir su visión, misión y objetivos.

- Eficiencia como coherencia entre los recursos que se invierten, el esfuerzo y tiempo requerido con el logro de los objetivos que se desean alcanzar.

Otro planteamiento identifica que existen tres tipos de definiciones para explicar el concepto de calidad o cualquier otro. Las definiciones estipulativas, las descriptivas y las programáticas. La primera de ellas incluye argumentos concretos sobre el significado que quiere dársele a un término en un contexto (son utilizadas principalmente en los aspectos legales). El segundo grupo trata de explicar ¿qué es? el objeto de la definición e incluye 
la comparación de sus características. Por último, las programáticas son meramente prácticas e incluyen opiniones (Olaskoaga, Espinosa y Partida, 2015). Los autores concluyen que las definiciones de calidad en su mayoría son programáticas, ya que presentan contenidos y enfoques diferentes que responden a posturas distintas, de acuerdo a los actores que participan en las instituciones de educación superior.

\subsection{LA PERSPECTIVA INTRÍNSECA Y EXTRÍNSECA DE LA CALIDAD}

La calidad también puede analizarse desde dos grandes perspectivas: intrínseca y extrínseca. La primera de ellas tiene que ver con el abordaje propio de la disciplina o ciencia que se analiza, planteando que solo los expertos de dicha área específica del conocimiento pueden emitir comentarios $\mathrm{u}$ opiniones sobre su calidad. Se establece que el conocimiento tiene valor en sí mismo y que es independiente al que aprende, al proceso de enseñanza y aprendizaje e incluso al contexto. La dimensión extrínseca por su parte es mucho más amplia, ya que aborda la calidad desde diversos puntos de vista emitidos por los actores que intervienen en el proceso educativo. Esto hace más complejo poder establecer una definición única y consensuada de calidad (Casaliz, 1991).

El Consejo Nacional de Acreditación CNA (2006), concuerda con la visión intrínseca y extrínseca, ya que identifica dos componentes que influyen en el concepto de calidad. El primero tiene que ver con aspectos internos a la institución y el segundo con atributos del contexto global al que pertenece la universidad. Es por ello que en muchas ocasiones se reconoce que se tiene calidad a partir de su prestigio, de los recursos académicos y financieros con que cuenta, o de los resultados de alguna de sus funciones sustantivas. Desde la perspectiva externa se identifica a la calidad por el grado de satisfacción de los estándares establecidos, por colegios profesionales, sistemas de acreditación o empleadores.

Ante estas dos perspectivas, el CNA propone una aproximación integral, entendiendo por calidad «aquello que determina la naturaleza de algo, como aquello que hace de algo lo que ese algo es» (p. 25). Posteriormente, el mismo Consejo amplía su definición, estableciendo que calidad es la síntesis de características que permiten reconocer un programa académico específico o una institución de determinado tipo y hacer un juicio sobre la distancia relativa entre el modo como en esa institución o en ese programa académico se presta dicho servicio y el óptimo que corresponde a su naturaleza. 
A continuación, se presentan diversas aproximaciones al concepto de calidad desde la perspectiva extrínseca a la que se ha hecho referencia. De acuerdo a Silva, Reich y Vásquez (2003) citando a Harvey y Green se plantean las siguientes:

1. Como algo excepcional, distintivo, elitista o exclusivo; lo que conlleva a que solo un pequeño grupo o sector le sea posible alcanzarla. Son pocas las instituciones que logran entrar al club de la calidad. Bajo este punto de vista la calidad no requiere de criterios específicos, ya que se reconoce por olfato o instinto. Esta visión está estrechamente ligada al prestigio o reputación que las universidades han ido alcanzando a lo largo del tiempo, dejando por fuera indicadores que puedan medirse y permitan disminuir la subjetividad de este abordaje.

La calidad desde esta dimensión se confunde muchas veces con el concepto de excelencia y distinción de acuerdo a Bricall (2000), quien plantea también que se considera la calidad de una institución educativa superior por algunas de sus características: docentes, estudiantes, precios de sus cuotas de estudio, su historia, entre otras. ¿Es posible entonces que pueda garantizarse la calidad de una institución por su reputación social? ¿Qué no sea necesaria una evaluación objetiva que permita alcanzar conclusiones precisas? ¿Se puede plantear que la calidad es todo aquello que las personas creen que es?

2. Como perfección o consistencia, haciendo énfasis en la idea de ausencia de defectos, tratando de asegurar que las tareas se realizan de manera correcta desde el principio, asociándose intrínsecamente con la existencia de una amplia cultura de calidad, lo que implica la responsabilidad de todos y cada uno de los actores en la organización. Dentro del ámbito universitario es poco aplicable, debido a la complejidad y heterogeneidad de sus actores y tareas.

Desde esta perspectiva se compara el proceso de enseñanza aprendizaje con el proceso productivo de una empresa. ¿Es posible realizar una comparación de esta naturaleza? ¿Podrían asemejarse los insumos o materias primas, los procesos de transformación y los productos terminados con los estudiantes que ingresan a la universidad, su proceso de estudio y los egresados? Es necesario realizar un análisis riguroso que permita establecer si en un proceso educativo es viable lograr cero errores para reconocerlo como de calidad, ya que los elementos que intervienen son personas y no cosas, con mecanismos distintos de aprendizaje y contextos particulares.

3. La noción de transformación es otra forma de ver la calidad. Esta es una visión muy clásica y hace referencia al cambio cualitativo o valor agregado que ocurre en los estudiantes, en términos de conocimientos, valores y habilidades mediante el proceso educativo. Refleja el valor de la educación 
en sí misma, que transforma a la persona que tiene la posibilidad de acceder a ella. La medición de esta visión de calidad no es sencilla ni universal; ya que depende de lo que se defina por valor y es necesaria una minuciosa evaluación de las condiciones de entrada y de salida del estudiante.

Para Rodríguez (2013), citando a Asti, la noción de transformación es de las más significativas, ya que la verdadera calidad de una institución educativa radica en la capacidad de influir y transformar a los miembros de la comunidad, en especial a estudiantes y profesores. Toma como elemento principal el desarrollo personal y la formación integral de los alumnos desde que ingresan a la universidad hasta que se gradúan.

Esta transformación puede verse materializada por una serie de competencias generales y específicas, adquiridas por los profesionales egresados de las universidades y que conforman su perfil de egreso.

4. Desde el punto de vista económico, a la calidad se le asocia con costo beneficio, o dicho de otra forma con el retorno o rentabilidad que se logra respecto de la inversión que se realiza. Bajo esta óptica está presente la rendición de cuentas que la institución debe dar a sus usuarios y a quienes la sostienen. La efectividad implicada en esta aproximación se mide por medio de indicadores de desempeño y mecanismos de auditoría de la calidad.

Cobra especial importancia el análisis que los estudiantes realizan sobre el retorno de la inversión, los indicadores de inserción laboral y los ingresos que los graduados reciben por el trabajo que realizan. La calidad entonces dependerá de este tipo de indicadores.

Desde una perspectiva complementaria Sotomayor y Muga (2004), establecen que tanto en la noción de transformación como en la económica deben tomarse en cuenta la diferenciación entre la calidad de los programas académicos que ofrecen las instituciones de educación superior con la calidad de sus egresados. Luego de citar a varios autores llegan a la conclusión de que existen formas diversas de interpretar dicha calidad. Aunque socialmente se relaciona la calidad de un egresado con la de la institución donde realizó sus estudios, e incluso el atributo se otorga indistintamente, se debe tener presente que no necesariamente es así. La universidad sin lugar a dudas influye directamente en las competencias que los egresados adquieren y que luego se ven reflejadas en su desempeño profesional, pero existen otros factores a considerar como: a) características personales, b) capacitaciones previas y c) otras variables externas tanto al egresado como a la institución.

La calidad del programa académico o de la formación es la promesa básica que las instituciones ofrecen a sus aspirantes y estudiantes, así que la verificación de su cumplimiento debería ser la primera evaluación de calidad que se realice. Es obligatorio que se identifique que el valor agregado que 
se ofrece al momento del ingreso de los estudiantes se convierte en realidad al momento de la finalización de los estudios. Un enfoque de calidad como valor agregado permite medir la eficiencia y eficacia de las universidades. En definitiva, plantea Sotomayor y Muga que «la calidad de la institución puede ser entendida, desde esta perspectiva, como la capacidad de la entidad para entregar a un alumno el valor agregado prometido al costo acordado» (p. 22).

5. Se presenta también la visión de calidad como la adecuación o aptitud para el logro de los propósitos que la institución que se ha planteado, la que se asocia con la satisfacción o cumplimiento de las necesidades de la sociedad. La calidad se logra cuando se alcanzan los fines o propósitos para los que la institución fue creada. Esta se aleja de la noción excepcional, pues si algo no sirve al propósito esperado se vuelve irrelevante. Este punto de vista es más funcional ya que permite juzgar la calidad en relación a la forma en que una institución se ajusta a un propósito, pudiéndose así elaborar criterios específicos para su análisis. A la vez permite una evolución en la forma en que se entiende la calidad, ya que los propósitos de las organizaciones son susceptibles a revisiones y cambios cuando así se requiera.

De igual forma respeta la diversidad de la oferta educativa propia de cada sistema; permitiendo que cada una busque su nicho en la sociedad. Además, la misión y los objetivos deben considerar tanto las necesidades y expectativas de los usuarios actuales o potenciales, como la contribución que la propia institución pretende aportar a la sociedad a la que pertenece.

En ese sentido Orozco (2003), plantea que una universidad tiene calidad, cuando responde al fin para el que fue concebida desde sus orígenes. Señala que la universidad que se enfoca a la producción del conocimiento, a la búsqueda racional de la verdad como su interés primordial, se concibe de calidad.

Identificar la calidad como el logro del propósito o misión de una institución de educación superior ha sido muy aceptada. Castaño y García (2012), presentan la definición desarrollada por el Programa de Cooperación entre Instituciones de Educación Superior de la Unión Europea y América Latina (ALFA), como «el grado de ajuste de las acciones de una institución para poner en marcha las orientaciones que emanan desde su misión y propósitos institucionales» (p. 22). Incluyen como aspectos fundamentales que en el quehacer universitario el rumbo lo marcan los objetivos y finalidades institucionales.

Srikanthan y Dalrymple (2003) identifican que cada noción de calidad desarrollada por Harvey y Green tiene más aceptación en un grupo que en otro dentro de las comunidades educativas. Por ejemplo, los financistas de la educación superior se sentirían más cómodos con la noción de valor por el 
dinero, mientras que los estudiantes con la de excelencia. Los empleadores por su parte con la de adecuación a una finalidad, siempre y cuanto esta tenga presente la formación de profesionales calificados y aptos para los requerimientos del mercado laboral. Los académicos respaldarían la concepción de calidad como transformación, ya que es la que responden de mejor forma a la visión clásica de universidad.

\subsection{Nueva interpretación de las aproximaciones de calidad de Harvey Y GREEN}

Retomando la idea de que muchos de los conceptos de calidad que se han implementado en la educación superior han sido trasplantados del ámbito empresarial y que no siempre responden a su contexto, Olaskoaga, Barandiaran, Barrenetxea, Cardona y Mijangos (2011), desarrollaron una nueva interpretación de los planteamientos de Harvey y Green presentados en 1993. Este análisis trata de adaptarlos al ámbito de las instituciones de educación superior, relacionándolos a su vez con sus distintos actores. El primer paso que ejecutaron fue la operacionalización de los conceptos en afirmaciones que fueran más comprensibles y tuvieran un mayor significado en el ámbito académico (p. 48).

C1. La calidad consiste en desarrollar las capacidades del estudiante para avanzar en su propia transformación.

C2. La calidad consiste en formar estudiantes capaces de asumir un compromiso social.

C3. La calidad consiste en el cumplimiento de los objetivos y la misión que ha establecido la institución.

C4. La calidad consiste en satisfacer las expectativas de todos los involucrados.

C5. La calidad consiste en satisfacer las expectativas de los alumnos y de los empleadores.

C6. La calidad consiste en el cumplimiento y la mejora de estándares establecidos.

C7. La calidad consiste en el conseguir la eficiencia: obtener los mejores resultados al menor costo.

Asimismo, elaboraron un mapa en la que sitúan los conceptos de calidad en dos dimensiones: el grado en que cada concepto de calidad se forma de acuerdo a un componente externo o interno y el grado en que representa una visión clásica o moderna de la gestión de la calidad. A continuación, se presenta la nueva perspectiva desarrollada: 
Figura 2. Los conceptos de calidad (Harvey y Green, 1993) Y LOS ÍTEMS DESARROLLADOS PARA OPERACIONALIZARLOS

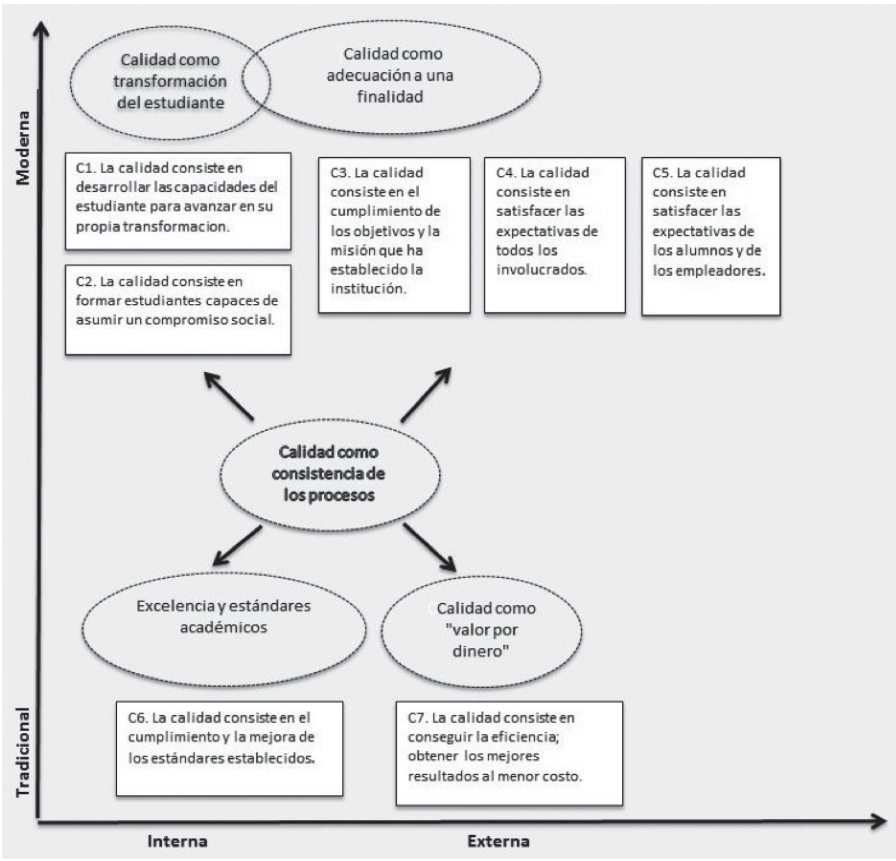

Fuente: Olaskoaga, Barandiaran, Barrenetxea, Cardona y Mijangos (2011, p. 49).

Es importante mencionar que el concepto de calidad como perfección o consistencia de los procesos se presenta desde una dimensión instrumental y transversal a las demás aproximaciones, ya que podría estar presente en las otras y apoyar su consecución. Esta nueva interpretación se adapta de mejor forma a la manera de ser de las instituciones de educación superior y hace posible una mejor comprensión de los distintos enfoques de la calidad.

\subsection{Abordajes adicionales DESDE La PERSPECTIVA EXTRÍNSECA DE LA CALIDAD}

Adicionalmente a los cinco enfoques presentados, Rodríguez Espinar (2013) incluye tres aproximaciones más al concepto de calidad desde la perspectiva extrínseca, que se desarrollan a continuación: 
1. Como adecuación al mercado laboral, poniendo el énfasis de la formación universitaria en satisfacer las necesidades del sector productivo del país. Se identifican como criterios importantes la adaptación de la oferta académica a la cantidad necesaria de graduados en las diferentes disciplinas, la inclusión de las competencias laborales identificadas por los distintos sectores en los planes de estudio y la flexibilidad de cambio y adaptación a las necesidades que surjan en los diversos campos laborales.

Este concepto genera posiciones encontradas, ya que existen grupos que no están de acuerdo en que sean necesidades externas las que rijan el quehacer universitario. A pesar de ello, no es desconocida la necesidad de que cada vez más exista una vinculación sólida entre la universidad y su entorno y la importancia de escuchar lo que los empleadores tiene que decir de la formación que se imparte a los profesionales que se insertan al mercado laboral.

2. Como satisfacción del usuario, que relaciona la calidad educativa con la calidad total en el ámbito de la empresa. Centra su atención como principal dimensión de calidad en la satisfacción de los requerimientos y expectativas de los usuarios actuales y potenciales. El principal reto que plantea, es el conocer con certeza lo que los estudiantes esperan de su formación y de los servicios que se les ofrecen; para poder así enfocarse en cumplir con dicha satisfacción. Se necesita un proceso permanente de identificación de requerimientos por parte del estudiantado y la medición del nivel de satisfacción que permita implementar acciones de mejora. Se hace fundamental también colocar como figura central del proceso al estudiante y sus necesidades, que muchas veces quiere mantenerse como figura pasiva.

3. Por último, se plantea la aproximación de calidad desde la perspectiva de la innovación educativa, cuyo enfoque se centra en la facilidad de adaptación de la estructura organizativa de la universidad a las necesidades actuales y las múltiples herramientas que se ofrecen, no solo en el ámbito tecnológico.

Aunque cada abordaje plantea aspectos importantes, se considera que aquellos que ven a la calidad desde la noción de transformación y como el logro de los propósitos o fines establecidos, son complementarios entre si y mucho más integrales. Cada organización puede responder desde alguno de estos enfoques a su concepción de calidad en los servicios que ofrece.

\subsection{OTRAS VISIONES DEL CONCEPTO DE CALIDAD}

Otras aproximaciones importantes al concepto de calidad fueron planteadas por Garvin (1988), clasificando las definiciones de acuerdo a 5 dimensiones: 
- Definiciones subjetivas y personales.

- Definiciones relacionadas con los productos o servicios que se ofrecen y sus atributos.

- Definiciones en relación a los usuarios y su satisfacción.

- Definiciones sobre el proceso de producción o al proceso de prestación del servicio.

- Definiciones que se plantean en relación al costo beneficio que se obtiene.

Lo expuesto anteriormente reafirma que la calidad se define de acuerdo al lente con el que se observa, convirtiéndose en una cuestión relativa y nunca absoluta. Dependerá entonces de la dimensión o dimensiones desde las que se analice. Comprender su significado se torna complejo, ya que cuenta con un sin número de enfoques y elementos que la conforman. De igual forma es posible concebirla como relativa, ya que las definiciones que cada institución desarrolla tienden a reflejar perspectivas diversas e incluir así, aspectos particulares de cada una; siendo totalmente válido.

Los autores continúan señalando que para que un sistema educativo se considere de calidad esta debe estar presente en cada elemento que lo conforma, es decir, se debe identificar la calidad en las personas, en los insumos, en los procesos, en los productos, en la infraestructura, en los procedimientos, en las técnicas pedagógicas y estrategias metodológicas de enseñanza, en los currículos y en la evaluación. En resumen, la calidad debe identificarse en cada una de las acciones que se desarrollen en una institución de educación superior.

Para Roa (2003) citando a Pabón, «la idea de calidad responde a necesidades y problemas muy particulares de las sociedades en momentos y situaciones específicas» (p. 171). Por lo tanto, los momentos que atraviesan las universidades y las regiones donde se encuentran ubicadas son tan diversos como los conceptos de calidad que manejan, ya que los contextos en los que se desenvuelven pueden ser totalmente distintos.

Por su parte Aguerrondo (2005) concibe la calidad en la educación como «la congruencia o consistencia entre los ejes fundamentales que conforman un sistema educativo» (p. 6). Dentro de ellos mencionan la coherencia que debe existir entre los ejes ideológicos, políticos, pedagógicos y la organización del aparato educativo. La calidad se da también cuando se puede identificar una relación pertinente entre el proyecto político del país y el proyecto educativo de la institución, haciendo énfasis en que el segundo debe impactar positivamente en el primero.

Márquez (2004) reafirma las definiciones de calidad educativa multidimensionales ya planteadas a lo largo de la presente investigación, 
considerando que muchos autores al referirse a ella solo la abordan desde una perspectiva que regularmente es la pedagógica. Establece entonces que está integrada por las dimensiones filosófica, pedagógica, económica, cultural y social. Al referirse al aspecto filosófico señala que un sistema educativo es de calidad cuando los contenidos que imparten son relevantes para un grupo social específico, respondiendo a sus necesidades. Desde la perspectiva pedagógica la calidad se logra cuando se alcanzan eficazmente las metas y objetivos trazados. Desde la económica, cuando los recursos asignados para su funcionamiento se utilicen con eficiencia; desde la cultural, cuando los contenidos y las estrategias de enseñanza concuerden con las necesidades de aprendizaje de la sociedad. La calidad entonces tiene que ver con el acceso equitativo de todos los grupos a la educación en sus distintos niveles.

Los acercamientos son diversos y se considera importante presentar el que desarrolla Bondarenco (2007) que tiene similitudes con Márquez, ya que enfatiza en una visión más filosófica de la calidad, determinando que muchos expertos orientan este tema desde la parte pragmática y de sus atributos. Explica su abordaje filosófico por medio de 15 características de la calidad:

1. Relativa, ya que depende de los aspectos que se evalúen y de la percepción y la satisfacción de los usuarios.

2. Continua, debido a que es muy difícil establecer el punto exacto donde se establece que algo es normal, empieza a ser de calidad o se convierte en excelente.

3. Subjetiva pues es relativa, debido a que involucra criterios de valor diferentes, asignados por personas diferentes. Lo que para alguien puede ser de calidad no necesariamente lo es para otro.

4. Social, ya que la calidad es un atributo socialmente determinado y varía de un grupo de personas a otro.

5. Dinámica bajo dos perspectivas, pues la calidad puede ser distinta de una cultura a otra y puede evolucionar y modificarse con el tiempo.

6. Participativa ya que, vista como un todo, son muchos factores lo que aportan a convertir algo como de calidad.

7. Axiológica, pues implica juicios de valor y apreciaciones positivas o negativas.

8. Evaluativa, debido a que está estrechamente ligada con el proceso de evaluación. La calidad siempre se está midiendo para determinar el nivel que posee e identificar qué aspectos son posibles de mejorar.

9. Dual, debido a que siempre hay dos lentes desde donde observarla. Puede ser como fabricante o prestador de servicios o bien como cliente o usuario. 
10. Sintética pues interviene toda una serie de variables, criterios e indicadores; muchas veces claramente definidos y otros casi imperceptibles.

11. Hipercompleja por la cantidad de conceptos que tratan de definirla.

12. Teleológica por el hecho de que lo bueno o de calidad es escaso y aplica para cosas muy concretas.

13. Utópica, pues se percibe como un ideal, una meta difícil de alcanzar.

14. Normativa, ya que debe responder a una serie de estándares o normas establecidos y que deben ser logrados.

15. Holística, pues a pesar de su descomposición en muchas partes, siempre se concibe como un todo.

Desde la perspectiva de la calidad total del servicio Arriel (1996), define el concepto de calidad en la educación superior como la integración de diversidad de vínculos que desarrolla la persona dentro del proceso global educativo y sus contextos socio-económicos, políticos y culturales específicos. La calidad entonces tiene que ver con las personas que conforman las universidades y sus capacidades cognitivas, laborales, éticas y ciudadanas, con el sistema educativo como tal, con los vínculos que sostiene con la comunidad en la que se desenvuelve, con la contribución al desarrollo del país, con su participación en el contexto internacional y con su capacidad de desarrollo científico y tecnológico; no solo para mejorar su quehacer internamente sino también para impactar en su entorno. Solo abarcando todos estos elementos, se puede entonces concebir que un sistema educativo puede aspirar a ser concebido como de calidad.

Como se ha venido señalando, Rodríguez Espinar (1991) establece que «no se da ni podrá darse una única concepción de calidad universitaria. El único posible nivel de concreción será el que se adopte a partir del enfoque evaluativo desde el que se evalúe dicha calidad» (p. 51).

Se ha abordado el concepto de calidad desde distintas perspectivas, identificando muchos factores que lo conforman y visiones complementarias. Es posible afirmar luego de los planteamientos de autores a lo largo de varias décadas, que el concepto de calidad es multidimensional, relativo, sistémico, construido histórica y colectivamente también; con matices políticos, ideológicos, pedagógicos y filosóficos, por mencionar algunos. Existen interpretaciones y definiciones como investigadores, que han profundizado en dicha temática. Es pues casi imposible encontrar un consenso que explique con toda claridad a que nos referimos cuando hablamos de calidad en la educación superior. 


\section{COMENTARIOS FINALES}

A pesar de ello, contar con una definición clara y consensuada de lo que se entiende por calidad en las instituciones de educación superior, es el punto de partida para su posterior evaluación. De igual forma hará posible el establecimiento de sistemas de garantía de calidad, que permitan la institucionalización de culturas de mejora continua. Es importante señalar que la concepción de la calidad puede variar de una institución a otra y adoptar matices distintos, pero tener claridad de lo que significa es indispensable para orientar así las acciones que sean necesarias para ofrecer servicios de excelencia; que a su vez propicien la regulación y la garantía y mejoramiento de la calidad. Las principales conclusiones obtenidas son:

1. A partir de la revisión realizada sobre el concepto de calidad se puede concluir que existe una gran cantidad de definiciones como autores, tratando de explicar de qué trata la calidad en la educación superior. Las discusiones concuerdan que no es posible llegar a una aproximación única, ya que es un concepto relativo. Por tal motivo, una universidad puede identificarse como de calidad en relación a una dimensión concreta y de menor calidad en relación a otra.

2. A pesar de todos los abordajes desde los que se analiza la calidad, pueden identificarse dos grandes perspectivas desde donde se interpreta: intrínseca y extrínseca. La primera señala que la calidad debe juzgarse desde lo interno de la propia universidad, mientras que la segunda es mucho más amplia, ya que aborda la calidad desde diversos puntos de vista emitidos por todos los actores que intervienen en el proceso educativo.

3. Las aproximaciones más aceptadas dentro de las universidades sobre el concepto de calidad son las de calidad como noción de transformación y como el logro de los propósitos o fines establecidos, ya que son complementarias entre sí. La calidad como propósito se logra cuando se alcanzan los fines para los que la institución fue creada. La de transformación hace referencia al cambio cualitativo o valor agregado que ocurre en los estudiantes por medio del proceso educativo; reflejando el valor de la educación en sí misma.

4. El recorrido llevado a cabo mediante la investigación de autores referente en el tema, llevan a afirmar que la calidad se define de acuerdo al lente con el que se observa, convirtiéndose en una cuestión relativa y nunca absoluta. Dependerá entonces de la dimensión o dimensiones desde las que se analice. Los autores señalan que para que un sistema educativo se considere de calidad, esta debe estar presente en cada elemento que lo conforma, es decir, se debe identificar la calidad en 
las personas, en los insumos, en los procesos, en los productos, en la infraestructura, en los procedimientos, en las técnicas pedagógicas y estrategias metodológicas de enseñanza, en los currículos y en la evaluación.

5. Ya que la calidad se define e interpreta desde distintos puntos de vista, contar con una definición clara y consensuada de lo que se entiende por calidad en las instituciones de educación superior, es el punto de partida para su posterior evaluación. De igual forma hará posible el establecimiento de sistemas de garantía de calidad, que permitan la institucionalización de culturas de mejora continua.

\section{REFERENCIAS}

Aguerrondo, I. (2005). La calidad en la educación: Ejes para su definición y evaluación. Calidad y Equidad en la Educación. Organización de Estados Iberoamericanos. Recuperado de: www.campus-oei/calidad/aguerrondo.htm

Ardila Rodríguez, M. (2011). Calidad de la educación superior en Colombia, ¿Problema de compromiso colectivo? Revista Educación y Desarrollo Social, 5, 44-55.

Arríen, J. B. (1996). Calidad y acreditación: Exigencias a la universidad. Documento de Trabajo Comisión, 2. Recuperado de:http://biblioteca.uahurtado.cl/ujah/reduc/ $\mathrm{pdf} / \mathrm{pdf} / 8156 . \mathrm{pdf}$

Bondarenko, N. (2007). Acerca de las definiciones de la calidad de la educación. Educere, (39), 613-621.

Bricall, J. M. (2000). Informe Universidad 2000. Recuperado de: https://www.observatoriuniversitari.org/es/files/2014/05/Bricall_JM-2000-Informe-Universidad-2000.pdf

Bruner, J. (2003). In Consejo Nacional de Acreditación (CNA) (Ed.), Aseguramiento de la calidad y nuevas demandas sobre la educación superior en América Latina. Educación superior, calidad y acreditación. tomo I. Colombia: Linotipia Bolívar.

Buendía Espinosa, A. (2007). El concepto de calidad: Una construcción en la educación superior. Reencuentro - Universidad Autónoma Metropolitana. Unidad Xochimilco, (50), 28-34.

Casaliz, P. (1991). Gestión estratégica y evaluación de calidad. En AA.VV. Primer Encuentro Interuniversitario Nacional sobre Evaluación de la Calidad, 51-56.

Castaño-Duque, G. y García-Serna, L. (2012). Una revisión teórica de la calidad de la educación superior en el contexto colombiano. Educación y Educadores, 15(2), 219-243.

CNA, Consejo Nacional de Acreditación. (2006). Lineamientos para la acreditación de programas. Bogotá, DC Colombia,

Duk, C. y Narvarte, L. (2008). Evaluar la calidad de la respuesta de la escuela a la diversidad de necesidades educativas de los estudiantes. REICE. Revista Electrónica Iberoamericana Sobre Calidad, Eficacia y Cambio En Educación, 6(2), 137-156. Recuperado de: https://repositorio.uam.es/handle/10486/661105 
Garbanzo, M. (2007). Calidad y equidad de la educación superior pública. Aspectos por considerar en su implementación. Revista Educación, 31(2), 11-27.

Garvin, D. A. (1988). Managing quality: The strategic and competitive edge. New York: Free Press.

Giraldo, U., Abad, D. y Díaz, E. (2003). Bases para una política de calidad de la educación superior. Educación superior, calidad y acreditación. tomo I. En Consejo Nacional de Acreditación (CNA) (Ed.), Colombia: Linotipia Bolívar.

González, L. y Espinoza, O. (2008). Calidad en la educación superior: Concepto y modelos. Calidad en la Educación, 28, 244-276.

Márquez Jiménez, A. (2004). Calidad de la educación superior en México. ¿Es posible un sistema eficaz, eficiente y equitativo? las políticas de financiamiento de la educación superior en los noventa. Revista Mexicana de Investigación Educati$v a, 9(21), 477-500$.

Morales, P. (2001). ¿Qué significa enseñar e investigar con calidad y equidad en la Educación Superior? Calidad y Equidad en la Educación Universitaria Católica. España: Universidad Pontificia Comillas. 19-102.

Olaskoaga, J., Barandiaran, M., Barrenetxea, M., Cardona, A. y Mijangos, J. (2011). La calidad en la educación superior: Aportaciones a un debate abierto. en: Calidad en la educación superior: ¿Qué modelo y en qué condiciones? la opinión del profesorado en Argentina, España y México. México: Editorial Universitaria. Universidad de Guadalajara.

Olaskoaga Larrauri, J., Espinosa, M. y Robles, M. (2015). La diversidad semántica y el carácter político de las nociones de calidad en la educación superior de México. Revista de la Educación Superior, 44(173), 85-102. doi:http://dx.doi. org/10.1016/j.resu.2015.04.003

Orozco Silva, L. (2003). In Consejo Nacional de Acreditación (CNA) (Ed.), La calidad de la universidad, más allá de toda ambigüedad. en: Educación superior, calidad y acreditación. tomo I. Colombia: Linotipia Bolívar.

Orozco Silva, L. (2010). Calidad académica y relevancia social de la educación superior en América Latina. Revista Iberoamericana de Educación Superior, 1(1), 24-36.

Real Academia Española (2005). Diccionario del estudiante. Madrid: Santillana.

Red Iberoamericana para la Acreditación de la Calidad de la Educación Superior. (2004). Glosario internacional RIACES de evaluación de la calidad y acreditación: Documento Madrid 2004. Madrid: Omán Editores.

Roa, A. (2003). In Consejo Nacional de Acreditación (CNA) (Ed.), Logros y retos de la acreditación en Colombia. en: Educación superior, calidad y acreditación. tomo II. Colombia: Linotipia Bolívar.

Rodríguez Espinar, S. (1991). Calidad universitaria: Un enfoque institucional y multidimensional. La Evaluación de las Instituciones Universitarias, 39-72.

Rodríguez, S. (2013). La evaluación de la calidad en la educación superior. España: Editorial Síntesis.

Silva, M., Reich, R. y Vázquez, C. (2003). Autoevaluación universitaria: Principios y mecanismos desde la experiencia. Francia. Editorial Columbus.

Sotomayor, A. y Muga, A. (2004). La diferenciación por calidad, ¿Cuáles son los límites? Revista Calidad en la Educación, 21, 47-60. 
Srikanthan, G. y Dalrymple, J. (2003). Developing alternative perspectives for quality in higher education. International Journal of Educational Management, 17(3), $126-136$

Toranzos, L. (2001). En la búsqueda de estándares de calidad. En programa de calidad y equidad. Organización De Estados Iberoamericanos Para La Educación, La Ciencia y La Cultura, Recuperado de: http://www.oei.es/calidad.htm

Tünnermann, C. (1999). Los desafíos de la universidad latinoamericana para los próximos años. Materiales De Clase, Curso IGLU-Región Países Andinos,

UNESCO. (1999). Proyecto de declaración mundial sobre la educación superior en el siglo XXI: Visión y acción sostenible, (1), 86-102.

Vizcarra, N., Boza, E. y Monteiro, E. (2011). La no neutralidad en la evaluación de la calidad y modelos de evaluación de la educación superior, casos de: Colombia, Argentina y Brasil. neutrality in quality evaluation and in models of higher education evaluation: The cases of Colombia, argentina and brazil. Avaliação: Revista Da Avaliação Da Educação Superior, 16(2), 291. doi:10.1590/S141440772011000200004 
